\title{
Selective Functionalization of 3-D Polymer Microstructures
}

\author{
Richard A. Farrer, ${ }^{\dagger}$ Christopher N. LaFratta,,$+\neq$ Linjie Li, ${ }^{\dagger, \ddagger}$ Julie Praino, $§$ Michael J. Naughton, ${ }^{\Uparrow}$ \\ Bahaa E. A. Saleh,§ Malvin C. Teich,§ and John T. Fourkas*,t, \\ Eugene F. Merkert Chemistry Center, and the Department of Physics, Boston College, Chestnut Hill, Massachusetts \\ 02467, Department of Chemistry \& Biochemistry, University of Maryland, College Park, Maryland 20742, and \\ Department of Electrical \& Computer Engineering, Boston University, Boston, Massachusetts 02215
}

Received December 9, 2005; E-mail: fourkas@umd.edu

Multiphoton fabrication is gaining prominence as a technique for the creation of 3-D microstructures of arbitrary complexity. The majority of the work in this field has focused on multiphoton absorption polymerization (MAP),${ }^{1-8}$ although for many microdevice applications, it is also desirable to be able to incorporate materials other than polymers. However, it has so far proven difficult to use multiphoton absorption in the creation of freestanding 3-D microstructures, or components thereof, composed of materials such as metals, metal oxides, or biomolecules. Here we present a simple and general scheme for the selective functionalization of 3-D polymeric microstructures fabricated with MAP, making it possible to deposit any of a wide range of materials on desired regions of these structures. We demonstrate the use of this technique for the creation of electrically conductive metal/polymer hybrid microdevices.

In MAP, an ultrafast laser is focused through a microscope objective into a prepolymer resin containing a photoinitiator that can be excited by the simultaneous arrival of two or more photons of near-infrared laser light. Because this process depends nonlinearly on the light intensity, at appropriate excitation energies, polymerization can be localized within the focal volume of the laser. Complex structures with submicron feature sizes are created by scanning the laser beam in a 3-D pattern. Once fabrication is complete, the unpolymerized resin is washed away with solvent, leaving behind the hardened structure.

Much of the work to date on MAP has focused on acrylic polymers, due to the broad commercial availability and high reactivity of acrylate monomers. A similarly wide variety of methacrylate monomers is available, but due to their significantly lower degree of reactivity, the use of these materials for MAP has been reported only once. ${ }^{9}$ We have found that at higher laser intensities it is possible to use MAP to create methacrylate microstructures that are of comparable quality to acrylate microstructures. Since acrylates and methacrylates can cross react, it is possible to use sequential MAP fabrication steps to create 3-D microstructures in which some portions are composed of acrylic polymer and others of methacrylic polymer. On the basis of the differential reactivity of these materials, it is possible to perform subsequent chemical reactions solely on the regions of the microstructures composed of one of the polymers.

A typical acrylic resin had two constituents, the trifunctional Sartomer SR368 and the pentafunctional Sartomer SR399, in a 1:1 weight ratio. ${ }^{7}$ The methacrylic resin was composed of Sartomer SR348, a difunctional monomer. In both cases, Lucirin TPO-L, which has strong two-photon absorption at $800 \mathrm{~nm},{ }^{7}$ was used as

$\dagger$ Eugene F. Merkert Chemistry Center, Boston College.

Eugene F. Merkert Chemis
University of Maryland.

\&oston University.

II Department of Physics, Boston College. the photoinitiator at a concentration of about $3 \mathrm{wt} \%$. Fabrication was performed with $100 \mathrm{fs}$ pulses from a Ti:sapphire oscillator with a center wavelength of $800 \mathrm{~nm}$ and a repetition rate of $76 \mathrm{MHz}$. A $40 \mathrm{X}$ oil-immersion objective with a numerical aperture of 1.3 was used to focus the laser into the sample. Typical fabrication powers and velocities were $10 \mathrm{~mW}$ and $10 \mu \mathrm{m} / \mathrm{s}$ for acrylates and $20 \mathrm{~mW}$ and $10 \mu \mathrm{m} / \mathrm{s}$ for methacrylates, respectively; all powers were measured outside of the microscope. In all cases, fabrication was performed on a glass substrate that had been modified with (3methacryloxypropyl) trimethoxysilane to promote adhesion of the polymer. ${ }^{7}$ The structures were washed in $\mathrm{N}, \mathrm{N}$-dimethylformamide and absolute ethanol after fabrication in each resin. Immediately following the final wash, the structures were immersed in a $20 \%$ $\mathrm{v} / \mathrm{v}$ solution of ethylenediamine in absolute ethanol for $1 \mathrm{~h}$, after which they were rinsed thoroughly in absolute ethanol. The diamine undergoes highly selective Michael addition with unreacted surface acrylates, leaving free amines on the surface of the acrylic portion of the structure but not on the methacrylic portion. ${ }^{10}$

Once amines are present on the desired polymer surface, any of a large number of chemical reactions can be used to deposit different materials on them. As examples, we discuss two different methods that can be employed to metallize the polymer systems presented here. The first method involves immersion of the amine-modified structures in a $1 \%$ aqueous solution of hydrogen tetrachloroaurate. Free amines lead to the reduction of gold ions onto the surface of the polymer, as is illustrated by the gold chain in Figure 1a. Note that because the glass substrate has been modified with methacrylates metal is not deposited on it.

The second method begins with treatment with $\mathrm{PdCl}_{2}$-saturated ethanol for $1 \mathrm{~h}$, leaving Pd complexed to the surface amines. Electroless deposition of any of a range of different metals can then be performed selectively on the acrylic polymer. Here we discuss electroless copper enhancement. ${ }^{11}$ The enhancement bath is prepared by dissolving $0.75 \mathrm{~g}$ of copper sulfate, $1 \mathrm{~g}$ of sodium hydroxide, and $3.5 \mathrm{~g}$ of sodium potassium tartrate in $25 \mathrm{~mL}$ of water. This solution is filtered just prior to use, and $2.5 \mathrm{~mL}$ of formaldehyde $(37 \%)$ is added with vigorous stirring. A small amount of this solution is dripped onto the polymer structure, and reaction is allowed to proceed for a short time. Enhancement is quenched via a Millipore water rinse. This process is followed visually on a microscope and can be repeated as many times as desired. Panels $b-e$ of Figure 1 demonstrate the selectivity of metallization using this procedure. The square on the left of Figure $1 \mathrm{~b}$ was created with methacrylate resin, and the adjacent square was created with acrylate resin. The metal has been deposited completely selectively on the acrylate square. Similarly, c-e of Figure 1 show an acrylic log-stack structure with a methacrylic structural frame. Once again, metallization has proceeded with complete selectivity on the acrylic portion of the structure. 


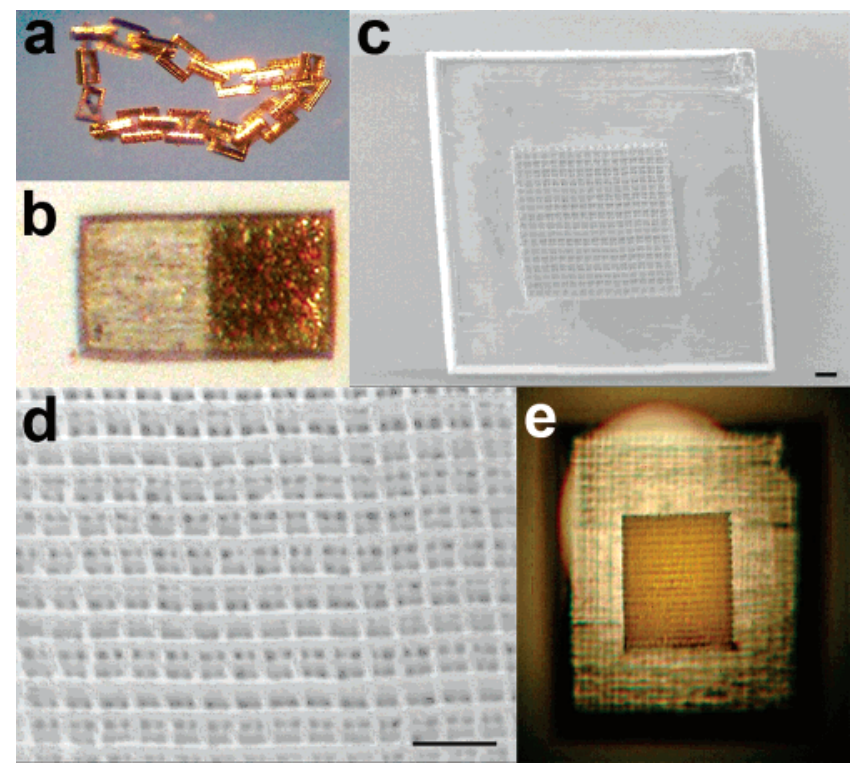

Figure 1. Selectively metallized microstructures created with MAP. (a) A gold-coated chain; each link is $70 \mu \mathrm{m}$ long. (b) Adjacent polymer squares that are $20 \mu \mathrm{m}$ on a side. The methacrylic square on the left is unfunctionalized, while the acrylic square on the right has been coated selectively with copper. (c and d) Scanning electron micrographs of a 3-D, acrylic log-stack structure. The surrounding frame is composed of a methacrylic polymer. Scale bars are $10 \mu \mathrm{m}$. (e) An optical micrograph of the same structure demonstrating that the logs have been selectively copper coated.

One impetus for selective metallization of polymeric microstructures is the incorporation of electrical functionality. To test the electrical conductivity of the metallized polymer, four-probe conductivity measurements were made on a variety of structures. Typical resistance values are in the range of $10 \Omega$ for metallized polymer leads that are on the order of $100 \mu \mathrm{m}$ long and $1 \mu \mathrm{m}$ wide. Improved conductivity can presumably be attained via an additional electrodeposition step.

An example of a selectively metallized, 3-D electrical structure that is difficult to fabricate with conventional techniques is the 16turn coil inductor shown in Figure 2. The supporting structure is composed of methacrylic polymer, and the inductor is composed of $\mathrm{Cu}$-coated acrylic polymer. The ideal geometrical inductance of this coil, with its $12 \mu \mathrm{m}$ radius, is about $1.1 \mathrm{nH}$. Using the same fabrication/metallization process, two pairs of conducting wires were deposited along with, and connected to, the inductor to facilitate electromagnetic measurements. We were able to characterize the complex impedance of this structure. It exhibits what appears to be a self-resonance that is manifested as a minimum in the impedance (Figure 2 inset) and as a sign change in the phase (not shown). The ideal geometrical inductance and capacitance of this coil are about $1 \mathrm{nH}$ and $2 \mathrm{fF}$, respectively, leading to an anticipated electromagnetic resonance frequency near $80 \mathrm{GHz}$. The observed resonance frequency is 6 decades lower than this prediction. On the other hand, the coil can also be considered a spring, for which we calculate a spring constant of order $0.00001 \mathrm{~N} / \mathrm{m}$, yielding a mechanical resonance in the $10 \mathrm{kHz}$ range. Thus, one possibility is that we are observing a capacitively-driven coil/spring mechanical resonance, a complex situation that warrants further investigation.

In conclusion, we have demonstrated the selective chemical functionalization of 3-D polymer microstructures and have used

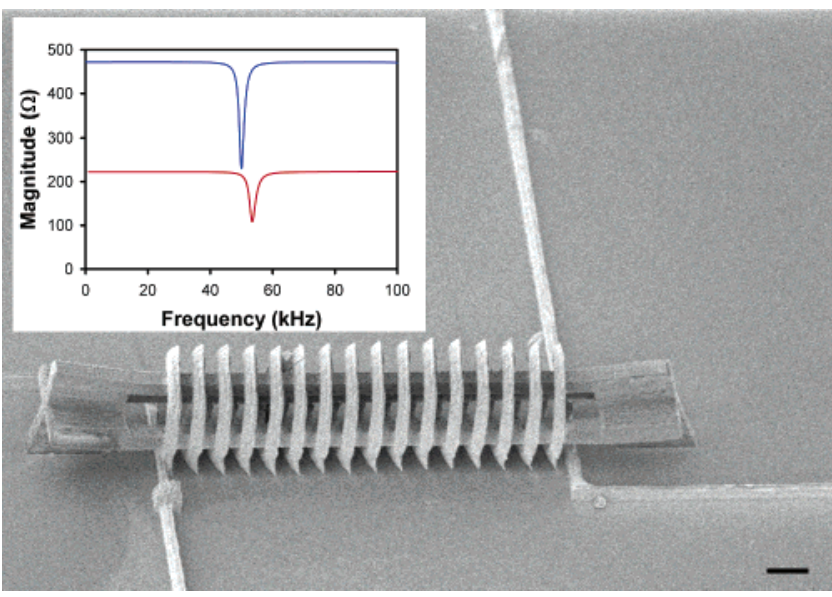

Figure 2. Electron micrograph of a copper-coated, 16-turn acrylic inductor with a methacrylic support. The scale bar is $10 \mu \mathrm{m}$. The inset shows the magnitude of the impedance of the inductor as a function of frequency. The red curve was obtained after additional metallization.

this technique to create a functional microinductor. We believe that it will be possible to increase the resonance frequency of such inductors by 3 to 4 orders of magnitude through optimization of materials and geometry. While we have limited our examples here to metallization, amines are highly versatile functional groups that can be used for the selective deposition and/or synthesis of many other materials, including biomolecules and metal oxides. In addition, this is but one example of a selective functionalization reaction for acrylic/methacrylic systems. Many other such reactions can be utilized with this and other combinations of photopolymer systems. This technique should open the door to using MAP for applications in areas, such as photonic crystals, metamaterials, and biosensing.

Acknowledgment. This work was supported by the National Science Foundation, Grant ECS-0210533.

\section{References}

(1) Maruo, S.; Nakamura, O.; Kawata, S. Opt. Lett. 1997, 22 (2), 132-134.

(2) Kawata, S.; Sun, H. B.; Tanaka, T.; Takada, K. Nature 2001, 412 (6848), 697-698.

(3) Witzgall, G.; Vrijen, R.; Yablonovitch, E.; Doan, V.; Schwartz, B. J. Opt. Lett. 1998, 23 (22), 1745-1747.

(4) Belfield, K. D.; Ren, X.; Van Stryland, E. W.; Hagan, D. J.; Dubikovsky, V.; Miesak, E. J. J. Am. Chem. Soc. 2000, 122 (6), 1217-1218.

(5) Cumpston, B. H.; Ananthavel, S. P.; Barlow, S.; Dyer, D. L.; Ehrlich, J. E.; Erskine, L. L.; Heikal, A. A.; Kuebler, S. M.; Lee, I.-Y. S.; McCordMaughon, D.; Qin, J.; Rockel, H.; Rumi, M.; Wu, X.-L.; Marder, S. R.; Perry, J. W. Nature 1999, 398, 51-54.

(6) Serbin, J.; Egbert, A.; Ostendorf, A.; Chichkov, B. N.; Houbertz, R.; Domann, G.; Schulz, J.; Cronauer, C.; Frohlich, L.; Popall, M. Opt. Lett. 2003, 28 (5), 301-303.

(7) Baldacchini, T.; LaFratta, C.; Farrer, R. A.; Teich, M. C.; Saleh, B. E. A.; Naughton, M. J.; Fourkas, J. T. J. Appl. Phys. 2004, 95 (11), 60726076.

(8) Baldacchini, T.; Fourkas, J. T. Three-Dimensional Nanofabrication Using Multiphoton Absorption. In Encyclopedia of Nanoscience and Nanotechnology; Schwarz, J. A., Contescu, C. I., Putyera, K., Eds.; Marcel Dekker: New York, 2004; pp 3905-3915.

(9) Belfield, K. D.; Schafer, K. J.; Liu, Y. U.; Liu, J.; Ren, X. B.; Van Stryland, E. W. J. Phys. Org. Chem. 2000, 13 (12), 837-849.

(10) Muh, E.; Marquardt, J.; Klee, J. E.; Frey, H.; Mulhaupt, R. Macromolecules 2001, 34 (17), 5778-5785.

(11) Hidber, P. C.; Helbig, W.; Kim, E.; Whitesides, G. M. Langmuir 1996, 12 (5), 1375-1380.

JA0583620 